\title{
Treatment and Observation of Advanced Carbon-based Nanomaterials by Slow Electrons
}

Eliska Materna Mikmekova ${ }^{1}$, Ivo Konvalina ${ }^{1}$, Ilona Mullerova ${ }^{1}$, Michael Lejeune ${ }^{2}$ and Tewodros Asefa ${ }^{3}$

${ }^{1}$ Institute of Scientific Instruments of the CAS, v. v. i., Brno, Jihomoravsky kraj, Czech Republic, ${ }^{2}$ Université de Picardie Jules Verne, Amiens, Picardie, France, ${ }^{3}$ Rutgers, The State University of New Jersey, Piscataway, New Jersey, United States

The development of new types of materials such as advanced carbon-based nanostructures also requires the development of new surface sensitive characterization techniques to fully unravel the materials' structures. With regards to "surface" sensitive materials, low energy electron microscopy can be a very powerful tool for precise study of these sensitive materials, see Figure 1, A. Modern commercial scanning electron microscopes enable imaging and analysis of samples by low energy electrons even at very high magnification.

In the case of carbon based nano-materials, we have to also take care about their possible non-conducting character (presence of $\mathrm{sp}^{3} \mathrm{C}-\mathrm{C}$ bonds or their doping by oxygen, nitrogen, etc.), which can cause the charging of the samples during electron irradiation. Luckily, at low landing energies, a charge balance could be achieved when an electron beam impinges on an insulating surface, at so-called critical energy: the number of impacted electrons equals the number of emitted electrons, as a result of which the charging effect is not observed. Figure 1, B shows the selected doped carbon nanostructures, where the ideal observation energy (critical energy) was discovered at $0.5 \mathrm{keV}$.

The low-energy-electron irradiation (treatment) can be also a powerful technique for the removal of adsorbed molecules and even polymer residues (PMMA) used for example for CVD graphene transfer. The most powerful electrons had an energy of about $500 \mathrm{eV}$, but the big impact of graphene quality was observed by Raman spectroscopy. According Raman spectroscopy, $50 \mathrm{eV}$ electron treatment is more appropriate for graphene cleaning. During $50 \mathrm{eV}$ irradiation, the polymer residues were successfully removed, and graphene quality kept its original state, see Figure 2. It should be noted, that electroninduced in-situ cleaning procedure can be gentle, experimentally convenient and very effective for wide range of specimens. [1,2]

The samples were studied by a unique method involving scanning low energy electron microscopy (SLEEM, SEM equipped with a cathode lens system - CL) and low voltage scanning transmission electron microscopy (LV STEM). Specifically a XHR S(T)EM Magellan 400 (FEI ${ }^{\circledR}$ ) microscope was used [3]. 

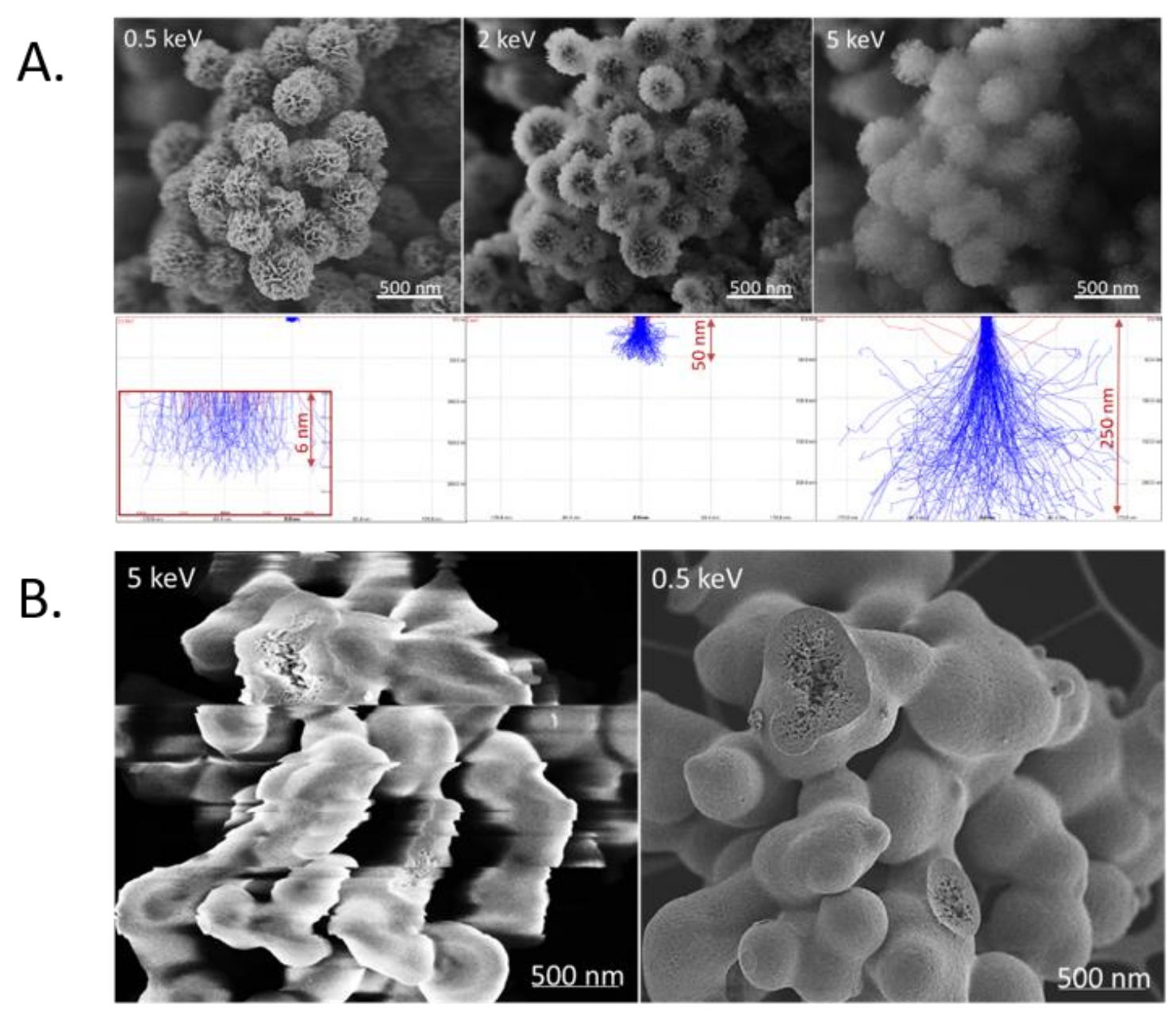

Figure 1. A: Surface sensitivity (nanoporous carbon-based materials). Monte Carlo simulations of interaction volumes at $0.5 \mathrm{keV}, 2 \mathrm{keV}$ and $5 \mathrm{keV}$ in the material. B: Charging elimination (heteroatomdoped metal carbonate-based nanostructures). 


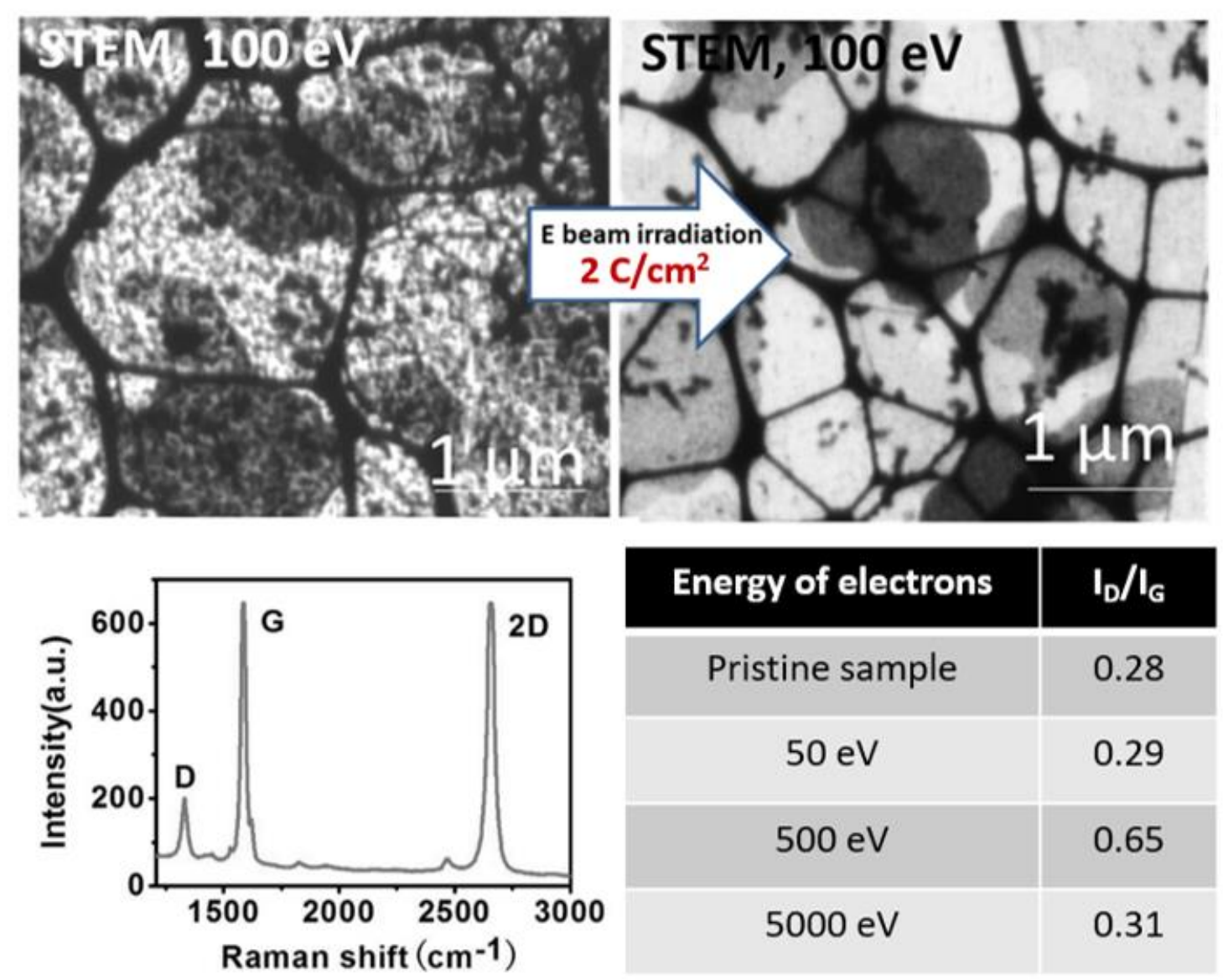

Figure 2. Treatment by slow electrons (graphene).

References

[1] L. Frank, E. Mikmeková, M. Lejeune, Applied Surface Science 407 (2017), p. 105

[2] E. M. Mikmeková et al., Journal of Electron Spectroscopy and Related Phenomena (2019), in press

[3] The authors acknowledge funding from the Technology Agency of the Czech Republic (Centre of Electron and Photonic Optics, no: TN01000008). 